\title{
Applying the Large Parameter Technique for Solving a Slow Rotary Motion of a Disc about a Fixed Point
}

\author{
A. I. Ismail (iD) 1,2 \\ ${ }^{1}$ Department of Mechanics, College of Engineering and Islamic Architecture, Umm Al-Qura University, Makkah, Saudi Arabia \\ P. O. Box 5555 \\ ${ }^{2}$ Mathematics Department, Faculty of Science, Tanta University, Tanta, P.O. Box 31527, Egypt
}

Correspondence should be addressed to A. I. Ismail; aiismail@uqu.edu.sa

Received 27 July 2020; Revised 20 August 2020; Accepted 27 August 2020; Published 16 September 2020

Academic Editor: Linda L. Vahala

Copyright (c) 2020 A. I. Ismail. This is an open access article distributed under the Creative Commons Attribution License, which permits unrestricted use, distribution, and reproduction in any medium, provided the original work is properly cited.

In this paper, the motion of a disk about a fixed point under the influence of a Newtonian force field and gravity one is considered. We modify the large parameter technique which is achieved by giving the body a sufficiently small angular velocity component $r_{0}$ about the fixed $z$-axis of the disk. The periodic solutions of motion are obtained in the neighborhood $r_{0}$ tends to 0 . This case of study is excluded from the previous works because of the appearance of a singular point in the denominator of the obtained solutions. Euler-Poison equations of motion are obtained with their first integrals. These equations are reduced to a quasilinear autonomous system of two degrees of freedom and one first integral. The periodic solutions for this system are obtained under the new initial conditions. Computerizing the obtained periodic solutions through a numerical technique for validation of results is done. Two types of analytical and numerical solutions in the new domain of the angular velocity are obtained. Geometric interpretations of motion are presented to show the orientation of the body at any instant of time $t$.

\section{Introduction}

In [1], the authors considered the limiting case for the motion of a rigid body about a fixed point in the Newtonian force field and gravity one. The small parameter technique is applied to solve this problem. The authors defined this parameter inversely proportional to the third component of the angular velocity which is assumed to be sufficiently high. The periodic solutions and their graphical representations are obtained and illustrated geometrically. In [2], the authors admitted the KBM technique for solving the problem of a rotating heavy solid about a fixed point under the influence of a gyrostatic moment. They assumed a small parameter as in [1] and found the analytical and numerical solutions for the body which moves under its gravity and a gyro moment about the minor or the major axis of the ellipsoid of inertia. In [3], Leshchenko and Ershkov presented a new type of solving procedure for Euler-Poisson equations (rigid body rotation over a fixed point) in the presence of some restricted conditions on the body angular velocity or the applied perturbing torques. The author in [4] gave the regular precession of an asymmetric rigid body acted upon by a uniform gravity field and magnetic one. He obtained the equations of motion of the body and reduced them to a quasilinear autonomous system. He found the solution to the problem and its geometric interpretation of motion. Nayfeh [5] presented some perturbation methods such as Poincare', the KBM, the Multiple scales, and averaging techniques for solving this kind of motion under certain conditions. The authors in [6] studied the rotating symmetric rigid body about a fixed point in the Newtonian force field in a case analogous to Kovalevskaya's problem. They described the motion of the body and derived its equations of motion and find the solution to the problem assuming Kovalevskaya's conditions.

All the previous approximated methods depend on a small parameter achieved inversely proportional to the sufficiently high angular velocity component. This study gives many applications in physics [7], gyros [8,9], astronomy, engineering, aerospace, and other sciences. 
In our problem, we assume a slow rotation (weak spin) of the body instead of a fast rotation (high spin). We introduce a large parameter inversely proportional to the weak spin about the $z$-axis instead of a small parameter inversely proportional to the high spin about that axis. So, we must apply the large parameter technique instead of the small one used in the previous works. The validation of our results will be given.

\section{Formulation of the Problem and Construction of the Periodic Solutions}

In this section, we formulate the problem of the motion of the body, deduce the equations of motion, and construct the periodic solutions:

2.1. Formulation of the Problem. Consider a rigid body of mass $(\mathrm{M})$ rotates around a fixed point $O$. Let the two coordinate frames $O X, O Y, O Z$ and $O x, O y, O z$ are fixed in the space and the body, respectively. Initially, let the body spin with a small speed angular velocity component $r_{0}$ about the $z$-axis. Suppose that $p_{2}$ and $\gamma_{2}$ are the generalized coordinates of the reduced quasilinear autonomous system [2]. Let $g$ be the gravity acceleration; $A, B, C$ are the principal moments of inertia of the body for the moving coordinates; the point $\left(x_{0}, y_{0}, z_{0}\right)$ is the mass center of the body, and $\underline{R}$ is the position vector of $O_{1}$ to $O$. Let $M_{1}$ and $M_{2}$ be the basic amplitudes for $p_{2}$ while $M_{3}$ is the basic amplitude for $\gamma_{2}$. Considering $\beta_{1}(\mu), \beta_{2}(\mu)$ and $\beta_{3}(\mu)$ are the deviations for $p_{2}$ and $\gamma_{2}$ from their initial basic amplitudes $M_{1}, M_{2}$, and $M_{3}$ respectively.

Let the moving $z$-axis make an angle $\theta_{0} \approx \pi / 2$ with the downward fixed $Z$-axis. This case of a slow spinning rotary body when the natural frequency $\omega=1$ gives the rotary motion of a disc which is excluded from the previous case $\gamma_{0}^{\prime \prime}=\cos \theta_{0} \approx 0$. We achieve a large parameter $0 \prec \prec \mu \prec \infty$ inversely proportional to the sufficiently small value of $r_{0}$. Assume that the disc rotates about the $z$-axis in the presence of a Newtonian force field [3] and a gravitational one. We reduce the equations of motion for this case to the following autonomous system of two degrees of freedom [4]:

$$
\begin{aligned}
& \ddot{p}_{2}+p_{2}=\mu^{-2} F\left(p_{2}, \dot{p}_{2}, \gamma_{2}, \dot{\gamma}_{2}, \mu^{-1}\right), \\
& \ddot{\gamma}_{2}+\gamma_{2}=\mu^{-2} \Phi\left(p_{2}, \dot{p}_{2}, \gamma_{2}, \dot{\gamma}_{2}, \mu^{-1}\right),
\end{aligned}
$$

where

$$
\begin{gathered}
p_{2}=p_{1}-\mu^{-1} x_{0}{ }^{\prime} \gamma_{0}^{\prime \prime} / b, \\
\gamma_{2}=\gamma-\mu^{-1} a \gamma_{0}^{\prime \prime} p_{2}, \\
F=F_{2}+\mu^{-1} F_{3}+\cdots, \quad(F \Phi), \mu=c \sqrt{\gamma_{o}^{\prime \prime}} / r_{0}, \\
F_{2}=F_{2}\left(p_{2}, \dot{p}_{2}, \gamma_{2}, \dot{\gamma}_{2}\right), \quad\left(F_{2} F_{3} \Phi_{2} \Phi_{3}\right), \cdot=d / d \tau,
\end{gathered}
$$

where

$$
\begin{aligned}
& A_{1}=(C-B) / A, \quad(A B C), a=A / C,(a b), x_{o}{ }^{\prime}=x_{o} / \ell,\left(x_{o}{ }^{\prime} y_{o}{ }^{\prime}\right), \\
& \ell^{2}=x_{o}{ }^{2}+y_{o}{ }^{2}, \quad k=3 C / R M \ell, p_{1}=p / c^{2},\left(p_{1} q_{1}\right), r_{1}=r / r_{0}, c=\sqrt{M g \ell / C},
\end{aligned}
$$

where the symbols like $(a b),(a b c)$, and $(a b c d)$ denote omitted equations and $p, q, r$, and $\gamma, \gamma^{\prime} \gamma^{\prime \prime}$ are the components of the angular velocity vector and the cosines direction of the unit vector $Z^{\wedge}$.

The system (2) has the following integral [4]:

$$
\gamma_{2}^{2}+\dot{\gamma}_{2}^{2}+2 \mu^{-1} \gamma_{0}^{\prime \prime}\left(a p_{2} \gamma_{2}-b \dot{p}_{2} \dot{\gamma}_{2}+s_{21}\right)=1-\gamma_{0}^{\prime \prime 2}
$$

where

$$
s_{21}=a\left(p_{20} \gamma_{20}-p_{2} \gamma_{2}\right)-b\left(\dot{p}_{20} \dot{\gamma}_{20}-\dot{p}_{2} \dot{\gamma}_{2}\right)
$$

where the quantities $p_{20}, \gamma_{20}, \dot{p}_{20}$, and $\dot{\gamma}_{20}$ are the initial values of the corresponding variables.

2.2. Construction of the Periodic Solutions. In this subsection, we use the large parameter technique [5] to construct the periodic solutions of system (2) when the disc rotates with slow velocity about the minor axis of the ellipsoid of inertia. Assume that

$$
A+B=C, \quad a+b=1 .
$$

Using (8) and the definition of $s_{21}$, we get

$$
s_{21}^{(0)}=0.5 M_{3} E[\cos \varepsilon-\cos (2 \tau-\varepsilon)]
$$

where $E=\sqrt{M_{1}^{2}+M_{2}^{2}}, \varepsilon=\tan ^{-1}\left(M_{2} / M_{1}\right)$ and $M_{1}$ and $M_{2}$ are constants.

Substituting (9) into (3), we get

$$
\begin{aligned}
F_{2}^{(0)}= & M_{1} L_{21}^{*} \cos \tau+M_{2} L_{21}^{*} \sin \tau+\cdots, \\
\Phi_{2}^{(0)}= & {\left[N_{21}^{*}-0.25 a\left(M_{1}^{2}-M_{2}^{2}\right)\right] M_{3} \cos \tau } \\
& -0.5 a M_{1} M_{2} M_{3} \sin \tau+\cdots, \\
F_{3}^{(0)}= & {\left[L_{31}^{*} M_{1}+L_{32}^{*}\left(M_{1}^{2}-M_{2}^{2}\right)+L_{33}^{*} M_{3}\right] \cos \tau } \\
& +\left[L_{31}^{*} M_{2}+2 L_{32}^{*} M_{1} M_{2}+L_{34}^{*} M_{3}\right] \sin \tau+\cdots,
\end{aligned}
$$

where

$$
\begin{aligned}
L_{21}^{*}= & 2 M_{3} x_{0}^{\prime}+0.5(a-b)\left(M_{2}^{2}-M_{1}^{2}-k M_{3}^{2}\right) \\
& +k\left(\gamma_{0}^{\prime \prime 2}-0.5 M_{3}^{2}\right), \\
N_{21}^{*}= & \left(a M_{1}^{2}+b M_{2}^{2}\right)+2 M_{3} x_{0}^{\prime} \\
& +k\left[\gamma_{0}^{\prime \prime 2}+M_{3}^{2}(b-a)\right],
\end{aligned}
$$




$$
\begin{aligned}
L_{31}^{*}= & 2 \gamma_{0}^{\prime \prime}\left[-a^{2} b^{-1} x_{0}^{\prime} M_{1}+b^{2} a^{-1} y_{0}^{\prime} M_{2}+a(1+b) k M_{1} M_{3}\right] \\
L_{32}^{*}= & -0.5 a \gamma_{0}^{\prime \prime} k M_{3} \\
L_{34}^{*}= & 3 a^{-1} b^{-1} x_{0}^{\prime} y_{0}^{\prime} \gamma_{0}^{\prime \prime} . \\
L_{33}^{*}= & \gamma_{0}^{\prime \prime}\left\{-\left(2+a b^{-1}\right) b^{-1} y_{0}^{\prime 2}+\left(1+a^{-1}\right) a^{-1} y_{0}^{\prime 2}\right. \\
& \left.+a k\left[0.5 k M_{3}^{2}-M_{1}^{2}-M_{2}^{2}\right]\right\}
\end{aligned}
$$

Let

$$
\begin{aligned}
& g_{k}(\tau)=\int_{0}^{\tau} F_{k}^{(0)}\left(t_{1}\right) \sin \left(\tau-t_{1}\right) d t_{1} \\
& h_{k}(\tau)=\int_{0}^{\tau} \Phi_{k}^{(0)}\left(t_{1}\right) \sin \left(\tau-t_{1}\right) d t_{1}
\end{aligned}
$$

Using (15), (11), and (14), we get

$$
\begin{aligned}
& g_{2}(2 \pi)=-\pi M_{2} L_{21}^{*}, \\
& \dot{g}_{2}(2 \pi)=\pi M_{1} L_{21}^{*}, \\
& h_{2}(2 \pi)=0.5 \pi a M_{1} M_{2} M_{3}, \\
& \dot{h}_{2}(2 \pi)=\pi\left[N_{21}^{*}-0.25 a\left(M_{1}^{2}-M_{2}^{2}\right)\right] M_{3}, \\
& g_{3}(2 \pi)=-\pi\left[L_{31}^{*} M_{2}+2 L_{32}^{*} M_{1} M_{2}+L_{34}^{*} M_{3}\right], \\
& \dot{g}_{3}(2 \pi)=-\pi\left[L_{31}^{*} M_{1}+L_{32}^{*}\left(M_{1}^{2}-M_{2}^{2}\right)+L_{33}^{*} M_{3}\right] .
\end{aligned}
$$

Using (16) and McLaren's expansion in the form [9]:

$$
\begin{array}{r}
U=u+\frac{\partial u}{\partial M_{1}} \beta_{1}+\frac{\partial u}{\partial M_{2}} \beta_{2}+\frac{\partial u}{\partial M_{3}} \beta_{3} \\
+\frac{1}{2} \frac{\partial^{2} u}{\partial M_{1}^{2}} \beta_{1}^{2}+\cdots,\left\{\begin{array}{c}
U=G_{k}, H_{k} \\
u=g_{k}, h_{k}
\end{array}\right\},
\end{array}
$$

when $M_{1}=M_{2}=0$, we get

$$
\begin{aligned}
G_{2}(2 \pi)= & -\pi \beta_{2}\left[2 \tilde{M}_{3} x_{0}^{\prime}+0.5(a-b)\left(\beta_{2}^{2}-\beta_{1}^{2}-k \tilde{M}_{3}^{2}\right)\right. \\
& \left.+k\left(\gamma_{0}^{\prime \prime 2}-0.5 \tilde{M}_{3}^{2}\right)\right], \\
\dot{G}_{2}(2 \pi)= & \pi \beta_{1}\left[2 \tilde{M}_{3} x_{0}^{\prime}+0.5(a-b)\left(\beta_{2}^{2}-\beta_{1}^{2}-k \tilde{M}_{3}^{2}\right)\right. \\
& \left.+k\left(\gamma_{0}^{\prime \prime 2}-0.5 \tilde{M}_{3}^{2}\right)\right], \\
G_{3}(2 \pi)= & -2 \pi \beta_{2} \gamma_{0}^{\prime \prime}\left[b^{2} a^{-1} y_{0}^{\prime} \beta_{2}-a^{2} b^{-1} x_{0}^{\prime} \beta_{1}+a(1+b) k \beta_{1} \tilde{M}_{3}\right] \\
& +\pi \tilde{M}_{3} \gamma_{0}^{\prime \prime}\left[a k \beta_{1} \beta_{2}-3 a^{-1} b^{-1} x_{0}^{\prime} y_{0}^{\prime}\right],
\end{aligned}
$$

$$
\begin{aligned}
\dot{G}_{3}(2 \pi)= & \pi \gamma_{0}^{\prime \prime}\left\{2 \beta_{1}\left[b^{2} a^{-1} y_{0}^{\prime} \beta_{2}-a^{2} b^{-1} x_{0}^{\prime} \beta_{1}+a(1+b) k \beta_{1} \tilde{M}_{3}\right]\right. \\
& +\tilde{M}_{3}\left[a^{-2}(a+1) y_{0}^{\prime 2}-b^{-2}(b+1) x_{0}^{\prime 2}\right. \\
& \left.\left.+0.5 a k\left(k \tilde{M}_{3}^{2}-3 \beta_{1}^{2}-\beta_{2}^{2}\right)\right]\right\}, \\
H_{2}(2 \pi)= & 0.5 a \pi \beta_{1} \beta_{2} \tilde{M}_{3}, \\
\dot{H}_{2}(2 \pi)= & \pi \tilde{M}_{3}\left[2 x_{0}^{\prime} \tilde{M}_{3}-\left(a \beta_{1}^{2}+b \beta_{2}^{2}\right)-0.25 a\left(\beta_{1}^{2}-\beta_{2}^{2}\right)\right. \\
& \left.+k \gamma_{0}^{\prime \prime 2}+k \tilde{M}_{3}^{2}(b-a)\right],
\end{aligned}
$$

where $\tilde{M}_{i}=M_{i}+\beta_{i}, i=1,2,3$.

Using (18), the independent periodicity conditions give [10]

$$
\begin{aligned}
v_{0} \beta_{1} & =-\mu^{-1} v_{1}+\cdots, \\
v_{0} \beta_{2} & =-\mu^{-1} v_{2}+\cdots, \\
\alpha\left(\mu^{-1}\right) & =\mu^{-2} \pi\left[2 M_{3} x_{0}^{\prime}+k\left(\gamma_{0}^{\prime \prime 2}+C_{1} M_{3}^{2}\right)\right]+\cdots,
\end{aligned}
$$

where

$$
\begin{aligned}
& v_{0}=a_{1} \beta_{1}^{2}+a_{2} \beta_{2}^{2}-0.5 k\left(C_{1}+1\right) M_{3}^{2}, \\
& a_{1}=0.5(b+0.67 a), \\
& a_{2}=0.5(b+0.5 a), \\
& v_{1}=M_{3} \gamma_{0}^{\prime \prime}\left[2 a^{-2}(a+1) \gamma_{0}^{\prime 2}-2 b^{-2}(b+1) x_{0}^{\prime 2}+a k^{2} M_{3}^{2}\right], \\
& v_{2}=-6 M_{3} \gamma_{0}^{\prime \prime} a^{-1} b^{-1} x_{0}^{\prime} y_{0}^{\prime} .
\end{aligned}
$$

The expansions $\beta_{1}, \beta_{2}$ are put in power series form as follows:

$$
\begin{aligned}
& \beta_{1}=\sum_{k=1}^{3} \mu^{-k} \ell_{k}+O\left(\mu^{-4}\right), \\
& \beta_{2}=\sum_{k=1}^{3} \mu^{-k} m_{k}+O\left(\mu^{-4}\right) .
\end{aligned}
$$

Substituting (22) into (19) and equating the coefficients of $\mu^{-1}$ in both sides, we get

$$
\begin{aligned}
& \ell_{1}=2 v_{1}\left[k\left(C_{1}+1\right) M_{3}^{2}\right]^{-1}, \\
& m_{1}=2 v_{2}\left[k\left(C_{1}+1\right) M_{3}^{2}\right]^{-1} .
\end{aligned}
$$


From [1] we get

$$
\begin{aligned}
& q_{1}=-\dot{p}_{1}+\mu^{-1} a^{-1}\left(y_{0}^{\prime} \gamma_{0}^{\prime \prime}-z_{0}^{\prime} \gamma^{\prime}+a k \gamma^{\prime} \gamma_{0}^{\prime \prime}\right)+\cdots, \\
& \gamma^{\prime}=\dot{\gamma}-\mu^{-1} \gamma_{0}^{\prime \prime} \dot{p}_{1}+\cdots .
\end{aligned}
$$

The following quantities are derived:

$$
\begin{aligned}
& M_{3}=1, \\
& \beta_{3}=-a \Gamma \mu^{-2} \tilde{M}_{1}-0.5 \mu^{-2} \Gamma^{2}+\cdots .
\end{aligned}
$$

Making use of (18), (23), (8), (24), (3), and (25), we obtain the following periodic solutions forms:

$$
\begin{aligned}
p_{1}= & v_{1} k^{*} \cos \tau+v_{2} k^{*} \sin \tau+\mu^{-1}\left[x_{0}^{\prime} b^{-1} \gamma_{0}^{\prime \prime}\right]+\cdots \\
q_{1}= & v_{1} k^{*} \sin \tau-v_{2} k^{*} \cos \tau+\mu^{-1}\left[y_{0}^{\prime} a^{-1} \gamma_{0}^{\prime \prime}-k \gamma_{0}^{\prime \prime} \sin \tau\right]+\cdots \\
r_{1}= & -0.25 \mu^{-2}\left[k C_{1}+4 x_{0}^{\prime}(1-\cos \tau)+4 y_{0}^{\prime} \sin \tau\right. \\
& \left.-k C_{1} \cos 2 \tau\right]+\cdots \\
\gamma= & \cos \tau+\mu^{-1} a \gamma_{0}^{\prime \prime}\left[v_{1} k^{*} \cos \tau+v_{2} k^{*} \sin \tau\right]+\cdots \\
\gamma^{\prime}= & -\sin \tau+\mu^{-1} b \gamma_{0}^{\prime \prime}\left[v_{1} k^{*} \sin \tau-v_{2} k^{*} \cos \tau\right]+\cdots \\
\gamma^{\prime \prime}= & \gamma_{0}^{\prime \prime}+\mu^{-2} \gamma_{0}^{\prime \prime} b^{-1} x_{0}^{\prime}(1-\cos \tau)+a^{-1} y_{0}^{\prime} \sin \tau \\
& -0.25 k(1-\cos 2 \tau)]+\cdots
\end{aligned}
$$

The correction of the period is:

$$
\begin{array}{r}
\alpha\left(\mu^{-1}\right)=\mu^{-2} \pi\left[2 x_{0}^{\prime}+\left(\gamma_{0}^{\prime \prime 2}+C_{1}\right) / b k *\right]+\cdots, \\
k=1 / b k^{*}, \tau=r_{o}{ }^{-1} t .
\end{array}
$$

\section{Geometric Interpretation of the Motion}

In this section, we search the description of the motion of the disk at any instant of the time using Euler's angles $\theta$, $\psi, \phi[11,12]$ :

$$
\begin{aligned}
\theta= & \theta_{0}-\mu^{-2} \cot \theta_{0}\left[\theta_{2}\left(t+t_{0}\right)-\theta_{2}\left(t_{0}\right)\right]+\cdots \\
r_{0}\left(\psi-\psi_{0}\right)= & 0.5 c \operatorname{cosec} \theta_{0}\left\{c \cot \theta_{0} k^{* 2}\left(v_{1}^{2}+v_{2}^{2}\right) t\right. \\
& +\operatorname{cosec} \theta_{0}\left[k \gamma_{0}^{\prime \prime} r_{0} t+\psi_{1}\left(t+t_{0}\right)-\psi_{1}\left(t_{0}\right)\right] \\
& \left.+2 \mu^{-1} \cot \theta_{0}\left[\psi_{2}\left(t+t_{0}\right)-\psi_{2}\left(t_{0}\right)\right]\right\}+\cdots \\
\phi-\phi_{0}= & {\left[r_{0}-0.5 c \cot ^{2} \theta_{0}\left(k-c r_{0}^{-1} k^{* 2}\left(v_{1}^{2}+v_{2}^{2}\right)\right)\right] t } \\
& -\mu^{-1} \cot \theta_{0} \operatorname{cosec} \theta_{0}\left[\phi_{1}\left(t+t_{0}\right)-\phi_{1}\left(t_{0}\right)\right]+\cdots
\end{aligned}
$$

where

$$
\begin{aligned}
\theta_{2}(t)= & -b^{-1} x_{0}^{\prime} \cos r_{0} t+a^{-1} y_{0}^{\prime} \sin r_{0} t+0.25 k \cos 2 r_{0} t, \\
\psi_{1}(t)= & 2 a^{-1} y_{0}^{\prime} \gamma_{0}^{\prime \prime} \cos r_{0} t-v_{2} k^{*} \cos 2 r_{0} t \\
& +\left(v_{1} k^{*}-0.5 k \gamma_{0}^{\prime \prime}\right) \sin 2 r_{0} t, \\
\psi_{2}(t)= & x_{0}^{\prime}(1-a)^{-1} \sin r_{0} t+0.5 C_{1} k^{* 2} v_{1} v_{2} \cos 2 r_{0} t \\
& -0.25 C_{1} k^{* 2}\left(v_{1}^{2}-v_{2}^{2}\right) \sin 2 r_{0} t, \\
\phi_{1}(t)= & a^{-1} y_{0}^{\prime} \gamma_{0}^{\prime \prime} \cos r_{0} t-0.5 v_{2} k^{*} \cos 2 r_{0} t \\
& +0.5\left(v_{1} k^{*}-0.5 k \gamma_{0}^{\prime \prime}\right) \sin 2 r_{0} t .
\end{aligned}
$$

These formulas depend on four arbitrary constants $\theta_{0}$, $\psi_{0}, \phi_{0}$, and $r_{0}$, where $r_{0}$ is sufficiently small. The obtained analytical periodic solutions are considered a generalization of the ones obtained in $[13,14]$.

\section{Numerical Solutions}

In this section, we use a smooth and suitable numerical method for obtaining the approximated numerical solutions for the autonomous system (2). Such a method is named the fourth-order Runge-Kutta method [15] which is used through a computerized program to find the numerical solutions of the considered problem. In the other side, we computerize the obtained analytical solutions and compare them with the numerical ones aiming to find the errors between them:

4.1. The Analytical Solutions. Rewriting the resulted analytical solutions in the form:

$$
\begin{aligned}
p_{2} & =v_{1} k^{*} \cos (\mathrm{h})+v_{2} k^{*} \sin (\mathrm{h}), \\
x & =d p_{2} / d t=-v_{1} k^{*} \sin (\mathrm{h})+v_{2} k^{*} \cos (\mathrm{h}), \\
\gamma_{2} & =\cos (\mathrm{h}), \quad y=d \gamma_{2} / d t=-\sin (\mathrm{h}) .
\end{aligned}
$$

Let the disk parameters are

$$
\begin{aligned}
A & =9, \\
B & =11, \\
C & =20, \\
x_{0} & =1, \\
y_{0} & =6, \\
R & =2000, \\
M & =200, \\
\gamma_{0}^{\prime \prime} & =0.000007, \\
\lambda & =0.6,
\end{aligned}
$$




$$
\begin{aligned}
& r_{0}=0.000013, \\
& T=18.78775142 .
\end{aligned}
$$

We obtain the following parameters of the motion:

$$
\begin{aligned}
C_{1} & =1.0 \mathrm{E}-01, \\
\ell & =6.082763, \\
x_{0}^{\prime} & =1.643990 E-01, \\
y_{0}^{\prime} & =9.863939 E-01, \\
g & =1.500000 E-07, \\
c & =3.020620 E-03, \\
\mu & =2.323554 E 06, \\
a & =4.5 E-01, \\
b & =5.5 E-01, \\
k & =2.465985 E-05, \\
\chi_{2} & =-1.726189 E-10 .
\end{aligned}
$$

Making use of (30), (31), and (32) through a computer program, we obtain the values of the analytical solutions (see Table 1).

4.2. The Numerical Solutions. The system (30) is rewritten in the form:

$$
\begin{aligned}
& \frac{d p_{2}}{d t}=x, \\
& \frac{d \gamma_{2}}{d t}=y, \\
& \frac{d x}{d t}=-p_{2}+\mu^{2} f_{1}, \\
& \frac{d y}{d t}=-\gamma_{2}+\mu^{2} g_{1},
\end{aligned}
$$

where $f_{1}=F_{2}, g_{1}=\Phi_{2}$.

Using (33), (31), (32), the fourth-order Runge-Kutta method, and the same initial values in the Table 1, we find the results of the numerical solutions in Table 2 . Table 1 is in good agreement with Table 2; that is, the analytical solutions are in full approximation with the numerical ones.

\section{Conclusions}

In this paper, we studied the singular value of the natural frequency problem $\omega=1$ in a limiting case when $\gamma_{o}^{\prime \prime}=$ $\cos \theta_{o} \approx 0$. In this case, the body rotates about the $z$-axis which should coincide with the minor axis of the ellipsoid of inertia. This problem corresponds to the disc motion
TABLE 1: The values of the analytical solutions in the interval $t \in[0,300]$.

\begin{tabular}{lcccc}
\hline$t$ & $p_{2 a}$ & $\gamma_{2 a}$ & $x_{a}=d p_{2 a} / d t$ & $y_{a}=d \gamma_{2 a} / d t$ \\
\hline 0 & 7.048539 & 1 & -2.028946 & 0 \\
10 & 4.52171 & 0.8102261 & -5.775176 & -0.5861173 \\
20 & $2.79 \mathrm{E}-01$ & 0.3129329 & -7.329452 & -0.9497752 \\
30 & -4.070127 & -0.3031333 & -6.101851 & -0.9529482 \\
40 & -6.874124 & -0.804146 & -2.558307 & -0.5944318 \\
50 & -7.069064 & -0.999947 & 1.956236 & -0.01030037 \\
60 & -4.580957 & -0.8162205 & 5.728294 & 0.5777406 \\
70 & $-3.54 \mathrm{E}-01$ & -0.3226997 & 7.326192 & 0.9465014 \\
80 & 4.007059 & 0.2933016 & 6.143451 & 0.95602 \\
90 & 6.847407 & 0.7979805 & 2.628977 & 0.6026833 \\
100 & 7.088839 & 0.9997878 & -1.883318 & 0.02059964 \\
110 & 4.639718 & 0.8221281 & -5.680805 & -0.5693026 \\
120 & $4.30 \mathrm{E}-01$ & 0.3324316 & -7.322155 & -0.9431273 \\
130 & -3.943567 & -0.2834384 & -6.1844 & -0.9589905 \\
140 & -6.819966 & -0.7917299 & -2.699373 & -0.6108714 \\
150 & -7.107862 & -0.9995226 & 1.810199 & -0.03089697 \\
160 & -4.697986 & -0.8279484 & 5.632714 & 0.5608044 \\
170 & $-5.05 \mathrm{E}-01$ & -0.3421288 & 7.317342 & 0.9396531 \\
180 & 3.879655 & 0.2735457 & 6.224691 & 0.961859 \\
190 & 6.791799 & 0.7853958 & 2.769477 & 0.6189939 \\
200 & 7.12613 & 0.9991513 & -1.736891 & 0.04119054 \\
210 & 4.755757 & 0.8336813 & -5.584021 & -0.5522458 \\
220 & $5.80 \mathrm{E}-01$ & 0.3517892 & -7.311752 & -0.9360793 \\
230 & -3.815332 & -0.263623 & -6.264326 & -0.9646258 \\
240 & -6.762911 & -0.7789784 & -2.839286 & -0.6270508 \\
250 & -7.143644 & -0.998674 & 1.663392 & -0.05148069 \\
260 & -4.813022 & -0.8393253 & 5.534739 & 0.5436295 \\
270 & $-6.56 \mathrm{E}-01$ & -0.3614123 & 7.305387 & 0.9324061 \\
280 & 3.750605 & 0.2536723 & 6.303295 & 0.9672902 \\
290 & 6.733307 & 0.7724778 & 2.908801 & 0.6350418 \\
300 & 7.160398 & 0.9980907 & -1.589723 & 0.06176443 \\
\hline & & & &
\end{tabular}

$A+B=C(a+b=1)$. The motion takes a slow spin rotation about the symmetric $z$-axis of the disc. The equations of motion and their first integrals are derived and reduced to two quasilinear differential equations of the second order and one first integral [16-19]. The periodic solutions for this problem are constructed applying the periodicity conditions and assuming a large parameter [20] proportional to $1 / r_{0}$. We used here the large parameter technique instead of the small one well-known in [21-26]. The advantage of this technique comes from the saving of the high initial energy which is given for the body to start the motion, and the solving of the problem in a new domain of the motion $F(t, \mu \longrightarrow \infty$, $\left.r_{o} \longrightarrow 0\right)$ and under new considerations. A geometric interpretation using Euler's angles of motion of the body as a function of time is presented. This interpretation shows the orientation of the disc at any instant of time $t$. There are numerical considerations of the problem and the solutions using the fourth-order Runge-Kutta method which is a 
TABLE 2: The values of numerical solutions in the interval $t \in[0,300]$.

\begin{tabular}{lcccc}
\hline$t$ & $p_{2 n}$ & $\gamma_{2 n}$ & $x_{n}=d p_{2 n} / d t$ & $y_{n}=d \gamma_{2 n} / d t$ \\
\hline 0 & 7.048539 & 1 & -2.028946 & 0 \\
10 & 4.521935 & 0.8102441 & -5.774878 & -0.5860706 \\
20 & 0.2793855 & 0.3130168 & -7.329234 & -0.9497204 \\
30 & -4.069078 & -0.3029831 & -6.102208 & -0.9529552 \\
40 & -6.87327 & -0.8039892 & -2.559511 & -0.5945567 \\
50 & -7.06908 & -0.9998797 & 1.954393 & -0.01054169 \\
60 & -4.582269 & -0.8163247 & 5.726515 & 0.5774587 \\
70 & -0.3566143 & -0.3229907 & 7.325408 & 0.9463065 \\
80 & 4.004261 & 0.292901 & 6.144369 & 0.9560346 \\
90 & 6.845462 & 0.7976249 & 2.63166 & 0.6029608 \\
100 & 7.088834 & 0.9996485 & -1.879637 & 0.02108086 \\
110 & 4.642086 & 0.8223141 & -5.677522 & -0.5687839 \\
120 & 0.4337946 & 0.3329276 & -7.320768 & -0.9427877 \\
130 & -3.939007 & -0.2827875 & -6.185843 & -0.9590072 \\
140 & -6.816898 & -0.7911727 & -2.703507 & -0.6112964 \\
150 & -7.107798 & -0.9993057 & 1.804683 & -0.0316163 \\
160 & -4.701379 & -0.8282109 & 5.627904 & 0.5600467 \\
170 & -0.5109161 & -0.3428261 & 7.315316 & 0.9391646 \\
180 & 3.873325 & 0.2726439 & 6.226625 & 0.9618729 \\
190 & 6.78758 & 0.7846335 & 2.775045 & 0.6195633 \\
200 & 7.125968 & 0.9988525 & -1.729537 & 0.04214693 \\
210 & 4.760143 & 0.8340154 & -5.577667 & -0.5512487 \\
220 & 0.5879716 & 0.3526854 & -7.309052 & -0.9354378 \\
230 & -3.807219 & -0.2624713 & -6.266709 & -0.9646314 \\
240 & -6.75751 & -0.7780079 & -2.846264 & -0.6277602 \\
250 & -7.143345 & -0.998288 & 1.654209 & -0.05267154 \\
260 & -4.818369 & -0.8397263 & 5.526817 & 0.5423905 \\
270 & -0.6649504 & -0.3625042 & 7.301974 & 0.9316074 \\
280 & 3.7407 & 0.2522707 & 6.306089 & 0.9672824 \\
290 & 6.726693 & 0.7712965 & 2.917158 & 0.6358864 \\
300 & 7.159923 & 0.9976127 & -1.578707 & 0.063189 \\
\hline & & & &
\end{tabular}

specialized and smooth method for finding the approximated periodic solutions of the nonlinear differential equations. Computerized programs are given as a validation of the technique and the results. These programs are carried in a closed interval of time $t \in[0,300]$ and showed that a full agreement between the analytical solutions and the numerical ones. The agreement of the numerical results with the analytical ones proves that the accuracy (validation) of the resulted solutions and studied techniques (see Tables 1 and 2). From the above tables, we find that the errors between the analytical and the numerical solutions are very small and can be neglected. To study the behavior of the body, we investigate a geometric interpretation of the motion using Euler's angles. We obtain an arbitrary initial angle of nutation $\theta_{o}$, precession $\psi_{o}$, and pure rotation $\varphi_{0}$. We note also that the expressions for the Euler's angles depend on four arbitrary constants $\theta_{0}, \psi_{0}, \phi_{0}$, and $r_{0}$ (where $r_{0}$ is sufficiently small). Moreover, we note that the disc spins slowly about the minor axis of the ellipsoid of inertia (that is a case of weak oscillations is obtained). In the first approximation, the case of a pseudoregular precession about the vertical axis is attained. As an example, the case of a regular Precession of a slowly spinning Lagrange gyroscope $\left(A=B, x_{0}=y_{o}=0\right)$ is obtained as a special case of this motion. There are many applications of these results in both military and civil life. This study is important for the satellite motion which has the correspondence of inertia moments, the antennas, the navigations, the solar collectors, and aerospace dynamics.

\section{Data Availability}

Data sharing is not applicable to this article as no datasets were generated or analyzed during the current study.

\section{Conflicts of Interest}

The author declares that he has no competing interests.

\section{Authors' Contributions}

I am the individual author of the manuscript.

\section{References}

[1] F. A. El-Barki and A. I. Ismail, "Limiting case for the motion of a rigid body about a fixed point in the Newtonian force field," ZAMM, vol. 75, no. 12, pp. 821-829, 1995.

[2] T. S. Amer, A. I. Ismail, and W. S. Amer, "Application of the Krylov-Bogoliubov-Mitropolski technique for a rotating heavy solid under the influence of a gyrostatic moment," Journal of Aerospace Engineering, vol. 25, no. 3, pp. 421-430, 2012.

[3] S. V. Ershkov and D. Leshchenko, "On a new type of solving procedure for Euler-Poisson equations (rigid body rotation over the fixed point)," Acta Mechanica, vol. 230, no. 3, pp. 871-883, 2019.

[4] H. M. Yehia, "On the regular precession of an asymmetric rigid body acted upon by uniform gravity and magnetic fields," Egyptian Journal of Basic and Applied Sciences, vol. 2, pp. 200205, 2015.

[5] A. H. Nayfeh, Perturbations Methods, WILEY-VCH Verlag $\mathrm{GmbH} \&$ co. KGaA, Weinheim, 2004.

[6] M. Iñarrea, V. Lanchares, A. I. Pascual, and A. Elipe, "Stability of the permanent rotations of an asymmetric gyrostat in a uniform Newtonian field," Applied Mathematics and Computation, vol. 293, pp. 404-415, 2017.

[7] M. M. Bhatti, R. Ellahi, A. Zeeshan, M. Marin, and N. Ijaz, "Numerical study of heat transfer and Hall current impact on peristaltic propulsion of particle-fluid suspension with compliant wall properties," Modern Physics Letters B, vol. 33, no. 35, article 1950439, 2019.

[8] L. Zhang, M. B. Arain, M. M. Bhatti, A. Zeeshan, and H. HalSulami, "Effects of magnetic Reynolds number on swimming of gyrotactic microorganisms between rotating circular plates filled with nanofluids," Applied Mathematics and Mechanics, vol. 41, no. 4, pp. 637-654, 2020.

[9] M. M. Bhatti, A. Shahid, T. Abbas, S. Z. Alamri, and R. Ellahi, "Study of activation energy on the movement of gyrotactic 
microorganism in a magnetized nanofluids past a porous plate," Processes, vol. 8, no. 3, p. 328, 2020.

[10] W. S. Amer, "On the motion of a flywheel in the presence of attracting center," Results in Physics, vol. 7, pp. 1214-1220, 2017.

[11] W. S. Amer, "The dynamical motion of a gyroscope subjected to applied moments," Results in Physics, vol. 12, pp. 14291435, 2019.

[12] T. S. Amer and W. S. Amer, "The rotational motion of a symmetric rigid body similar to Kovalevskaya's case," Iranian Journal of Science and Technology, Transactions A: Science, vol. 42, no. 3, pp. 1427-1438, 2018.

[13] T. S. Amer and W. S. Amer, "Substantial condition for the fourth first integral of the rigid body problem," Mathematics and Mechanics of Solids, vol. 23, no. 8, pp. 1237-1246, 2017.

[14] A. Gilat, "Numerical methods for engineers and scientists," Wiley, 2013.

[15] T. S. Amer, "The Dynamical Behavior of a Rigid Body Relative Equilibrium Position," Advances in Mathematical Physics, vol. 2017, Article ID 8070525, 13 pages, 2017.

[16] T. S. Amer, "On the dynamical motion of a gyro in the presence of external forces," Advances in Mechanical Engineering, vol. 9, no. 2, pp. 1-13, 2017.

[17] T. S. Amer, M. A. Bek, and M. K. Abouhmr, "On the vibrational analysis for the motion of a harmonically damped rigid body pendulum," Nonlinear Dynamics, vol. 91, no. 4, pp. 2485-2502, 2018.

[18] I. M. Abady and T. S. Amer, "On the application of KBM method for the 3-D motion of an asymmetric rigid body," Nonlinear Dynamics, vol. 89, pp. 1591-1609, 2017.

[19] T. S. Amer, "The rotational motion of the electromagnetic symmetric rigid body," Applied Mathematics \& Information Sciences, vol. 10, no. 4, pp. 1453-1464, 2016.

[20] A. I. Ismail, "Solving a problem of rotary motion for a heavy solid using the large parameter method," Advances in Astronomy, vol. 2020, Article ID 2764867, 7 pages, 2020.

[21] F. L. Chernousko, L. D. Akulenko, and D. D. Leshchenko, Evolution of motions of a rigid body about its center of mass, Springer, 2017.

[22] L. D. Akulenko, Y. S. Zinkevich, T. A. Kozachenko, and D. D. Leshchenko, "The evolution of the motions of a rigid body close to the Lagrange case under the action of an unsteady torque," Journal of Applied Mathematics and Mechanics, vol. 81, no. 2, pp. 79-84, 2017.

[23] A. I. Ismail and T. S. Amer, "The fast spinning motion of a rigid body in the presence of a gyrostatic momentum $\ell_{3}$," Acta Mechanica, vol. 154, no. 1-4, pp. 31-46, 2002.

[24] T. S. Amer, "On the motion of a gyrostat similar to Lagrange's gyroscope under the influence of a gyrostatic moment vector," Nonlinear Dynamics, vol. 54, no. 3, pp. 249-262, 2008.

[25] A. I. Ismail, T. S. Amer, S. A. el Banna, and M. A. el-Ameen, "Electromagnetic gyroscopic motion," Journal of Applied Mathematics, vol. 2012, Article ID 812645, 14 pages, 2012.

[26] L. D. Akulenko, D. D. Leshchenko, and F. L. Chernous'ko, "Perturbed motions of a rigid body, close to the Lagrange case," Journal of Applied Mathematics and Mechanics, vol. 43 , no. 5 , pp. 829-837, 1979. 